\title{
Quelle est la voie à suivre?
}

\section{Bruno Kesseli}

Dr med et lic. phil, rédacteur en chef

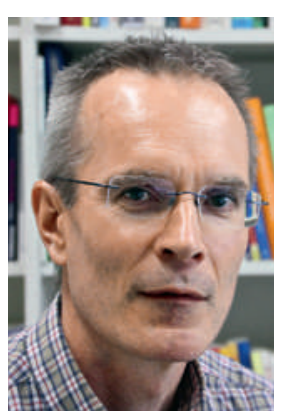

Nous sommes heureux, chère lectrice, cher lecteur, de vous voir consacrer un peu de temps à la lecture de ce magazine. Car chez les médecins - qui constituent la majorité de notre lectorat - le temps est une denrée rare, du moins à en croire notre expérience quotidienne (les autres lectrices et lecteurs ne sont peutêtre pas mieux servis). Cela n'en laisse que très peu pour la lecture de revues médicales spécialisées. Comme Corinna Schaefer, du Centre médical pour la qualité en médecine à Berlin, l'expliquait récemment lors d'un workshop [1], les médecins ne passent en moyenne que 20 minutes par semaine à l'étude de la littérature spécialisée. Même si une affirmation sommaire comme celle-ci doit être accueillie avec les précautions d'usage, ce temps est manifestement bien inférieur à ce que l'on s'imaginerait spontanément. Ce qui est vrai pour l'Allemagne ne devrait pas être fondamentalement différent en Suisse.

\section{Cela signifie-t-il que les médias imprimés classiques ont fait leur temps et disparaîtront complètement du marché à plus ou moins brève échéance?}

Dans ce contexte, les rédacteurs du Bulletin des médecins suisses voient-ils à terme un avantage dans le fait que le BMS n'est pas une revue spécialisée au sens d'un «Medical Journal»? Pas nécessairement, car d'après les enquêtes régulièrement menées par la WEMF [2], les médias imprimés en général et les revues grand public en particulier n'échappent pas à la tourmente. Au cours des dernières années, certains titres connus ont dû digérer une perte de leur lectorat d'un pourcentage à deux chiffres qui n'a pas pu être compensée par l'afflux de lecteurs inconditionnels du «on-line» désertant les médias imprimés ou nouvellement acquis. Cela signifie-t-il que les médias imprimés classiques ont fait leur temps et disparaîtront complètement du marché à plus ou moins brève échéance? Fait intéressant, les experts ne partent pas de l'hypothèse d'un tel scénario, comme me l'a récemment expliqué un membre du conseil d'administration d'un grand groupe de médias suisse. Bien sûr, il est généralement admis par les spécialistes que les médias papier «finiront par disparaître», mais les augures du journalisme semblent
Aux Editions médicales suisses, nous nous interrogeons naturellement sur la voie à suivre pour satisfaire au mieux les exigences et attentes de nos lectrices et lecteurs. Nous sommes aussi d'avis qu'il est indispensable de compléter la presse imprimée par des offres en

La haute importance que nous attachons aux journaux imprimés tient aussi à la diversité qualitative des différents médias.

ligne attirantes et conviviales. Le nouveau site Web de l'EMH entièrement reconçu vient d'être mis en ligne [3]. Au début du printemps, les sites Internet de nos grands magazines, dont le BMS, seront adaptés au «mobile responsive design» de manière à être aisément navigables et bien lisibles sur les smartphones et les tablettes.

La haute importance que nous attachons aux journaux imprimés tient aussi à la diversité qualitative des différents médias. Selon des études scientifiques dans le domaine du journalisme, la presse imprimée est particulièrement utile comme média rédactionnel lorsqu'il s'agit de transmettre des messages complexes et exigeants, un constat que les titres de presse traditionnels ont intégré depuis lors dans leur concept. Au lieu de privilégier la rapidité de la communication pour laquelle se battent désespérément les médias ultrarapides comme l'Internet et la télévision, ils ont mis la priorité sur la présentation des faits dans leur contexte et les informations de fond. Ce type de transmission de l'information correspond exactement au concept du BMS.

Vous êtes toujours là? Dans ce cas, je profite de cette occasion pour vous remercier de la fidélité que vous témoignez au BMS. Nous nous efforcerons de continuer à vous offrir une revue diversifiée et intéressante - sur papier ou en ligne, à choix. Je vous souhaite, chère lectrice, cher lecteur, de joyeuses fêtes et un bon départ dans la nouvelle année.

\section{Références}

1 Kesseli B. Wertvoll, aber aufwendig. Impressionen vom Workshop "Choosing wisely und Guidelines: Chancen und Stolpersteine». Bull Méd Suisses 2015;96(26):966-9.

2 WEMF AG für Werbemedienforschung. L'objectif statutaire de la société est la recherche systématique, continue et neutre sur les médias et la publicité en Suisse.

3 www.emh.ch 